\title{
Zitierweise und Siglen
}

Kapitelangaben im Inhaltsverzeichnis und den Titeln der Beiträge beziehen sich auf die Kapitelzählung der zweiten und dritten Auflage; eine vergleichende tabellarische Übersicht im Verhältnis zur ersten Auflage von Das Wesen des Christentums findet sich in der Einführung.

Bei den Zitaten werden Hervorhebungen einheitlich durch Kursivschrift wiedergegeben; editorische Zeichen (z.B. eckige Klammern oder Kursive zur Kennzeichnung von ergänzten Buchstaben) werden nicht wiedergegeben. In den Kommentaren und der Einleitung werden folgende Siglen verwendet:

GW Ludwig Feuerbach: Gesammelte Werke, hg.v. Werner Schuffenhauer, Berlin $1967 \mathrm{ff}$. (ab 1993 hg.v. der Berlin-Brandenburgischen Akademie der Wissenschaften durch W. Schuffenhauer), Bde. 1-14, 17-20.

FSW Ludwig Feuerbach: Sämtliche Werke, 10 Bde., hg.v. Wilhelm Bolin u. Friedrich Jodl, Stuttgart $1903 \mathrm{ff}$.

EC Ludwig Feuerbach: The Essence of Christianity, trans. G. Eliot, New York 1989.

PT Ludwig Feuerbach: Provisional Theses for the Reformation of Philosophy, trans. Daniel O. Dahlstrom, in: The Young Hegelians: An Anthology, ed. L.S. Stepelevich, Cambridge 1983, 156-171.

AA Immanuel Kant: Werke. Akademie-Ausgabe, Berlin $1900 \mathrm{ff}$.

HGW Georg Wilhelm Friedrich Hegel: Gesammelte Werke, Hamburg $1968 \mathrm{ff}$.

KGA Friedrich Daniel Ernst Schleiermacher: Kritische Gesamtausgabe, Berlin und New York (bzw. Boston), $1980 \mathrm{ff}$.

KpV Immanuel Kant: Kritik der praktischen Vernunft, in: AA 5, 1-163.

KrV Immanuel Kant: Kritik der reinen Vernunft, 1. Auflage (A) in: AA 4, 1-252, 2. Auflage (B) in: AA 3.

MEW Karl Marx und Friedrich Engels: Werke, Berlin $1956 \mathrm{ff}$.

MEGA Karl Marx und Friedrich Engels: Gesamtausgabe, Berlin $1975 \mathrm{ff}$. 
